Mathematical Research Letters 5, 305-325 (1998)

\title{
SPECTRAL TRANSFER AND POINTWISE ERGODIC THEOREMS FOR SEMI-SIMPLE KAZHDAN GROUPS
}

\author{
Amos Nevo
}

\begin{abstract}
Let $G$ be a connected semi-simple Lie group with finite center and no compact factors, and $(X, \mathcal{B}, m)$ a $G$-space with $\sigma$-finite $G$-invariant measure $m$. For each probability measure $\mu$ on $G$ consider the operator $\pi(\mu)$ : $L^{2}(X) \rightarrow L^{2}(X)$, given by $\pi(\mu) f=\int_{G} \pi(g) f d \mu(g)$. The explicit spectral estimates ("quantitative property T") of M. Cowling [Co2] and R. Howe $[\mathrm{H}]$ (see also $[\mathrm{H}-\mathrm{T}][\mathrm{Li}][\mathrm{Mo}][\mathrm{Oh}])$ are used to obtain explicit estimates of $\left\|\pi_{0}(\mu)\right\|$, where $\pi_{0}$ is the representation on the space orthogonal to the space of $G$-invariant functions, provided $\pi_{0}$ has a spectral gap. In particular, for actions of Kazhdan groups, the norm estimate is uniform and does not depend on the action. The norm estimates can be viewed as a spectral transfer principle, analogous to the transfer principle for amenable groups (see $[\mathrm{W}][\mathrm{Ca}][\mathrm{C}-\mathrm{W}][\mathrm{Hz} 1][\mathrm{Co} 3][\mathrm{Co} 4]$ ). The spectral estimates are used to derive exponential-maximal inequalities for natural families of averages on the group, as well as pointwise ergodic theorems in $L^{p}$ for these averages. The pointwise convergence of the averages to the ergodic mean is exponentially fast with an explicit rate. This phenomenon in the case of bi- $K$-invariant measures was established in [M-N-S], and here we discuss non-radial averages, which may be absolutely continuous, singular or discrete. Some other topics discussed are averages supported on lattice points, almost orthogonality, and best possible estimates of convolution norms and exponential rate of convergence to the ergodic mean.
\end{abstract}

\section{The spectral transfer principle for semi-simple Kazhdan Lie groups}

\subsection{Introduction.}

Let $G$ be a simple (non-compact) Lie group with finite center, not locally isomorphic to $S O(n, 1)$ or $S U(n, 1)$, or equivalently, satisfying property $T$ of Kazhdan [Ka] (see $\S 1.2$ for the definition). Then every strongly continuous unitary representation $\pi$ of $G$ which does not contain invariant unit vectors satisfies the following property : there exists a dense subspace of vectors $u, v$, whose matrix coefficient $\varphi_{u, v}(g)=\langle\pi(g) u, v\rangle$ belongs to $L^{p}(G)$, for some fixed $p=p(G)$, independent of $\pi$. This fundamental fact is due in general to M. Cowling [Co2, Th. 2.4.2, Th. 2.5.3], and for (irreducible) representations of the symplectic groups was proved independently by R. Howe [H, Th. 8.4] (see [H-T, Ch. V, §3] for a remarkable elementary proof for $S L_{n}(\mathbb{R})$, and [Oh] in general).

Received September 17, 1997.

Supported by the M. and M. L. Bank Mathematics Research Fund. 
It follows from Hölder's inequality that if $n \geq p / 2$, then $\pi^{\otimes n}$ has a dense set of matrix coefficients in $L^{2}(G)$, as noted in [Co2, Proof of lemme 3.3.2], [H, Prop. 6.1, Cor. 7.2] and [Mo, Prop. 3.5]. By [H, remark d), p. 285], or [M, Lemma 3.4] (see also [H-T, Ch. V, Cor. 1.2.4]), $\pi^{\otimes n}$ is equivalent to a subrepresentation of the sum of countably many copies of the regular representation of $G$ (denoted $\left.\infty \cdot \lambda_{G}\right)$. Furthermore, by [H, Prop. 6.4], for any closed unimodular subgroup $H \subset G,\left.\pi^{\otimes n}\right|_{H}$ has a dense set of matrix coefficients in $L^{p}(H)$, and hence $\left.\pi^{\otimes n}\right|_{H} \subset \infty \cdot \lambda_{H}$.

This discussion is summarized by the following statement, noted in various forms by M. Cowling, R. Howe and C.C. Moore, which we shall designate as

The spectral transfer principle. (see [Co2], $[\mathrm{H}],[\mathrm{Mo}])$ Let $G=\prod_{i=1}^{N} G_{i}$ be a connected semi-simple Kazhdan Lie group with finite center, none of whose simple factors $G_{i}$ is compact. Let $n=n(G)<\infty$ be the smallest even integer $n=2 k \geq \frac{1}{2} p(G)$. Then for every strongly continuous unitary representation $\pi$ of $G$ with no $G_{i}$-invariant unit vector (for all $1 \leq i \leq N$ ), $\pi^{\otimes n}$ can be embedded as a subrepresentation of $\oplus_{i=1}^{\infty} \lambda_{G}$, where $\lambda_{G}$ is the left regular representation. We write: $\pi^{\otimes n} \subseteq \infty \cdot \lambda_{G}$. Similarly $(\pi \otimes \bar{\pi})^{\otimes k} \subseteq \infty \cdot \lambda_{G}$, where $\bar{\pi}$ is the contregredient representation. Furthermore, for any closed unimodular subgroup $H \subset G,\left.\pi^{\otimes n}\right|_{H} \subset \infty \cdot \lambda_{H}$ and $\left.(\pi \otimes \bar{\pi})^{\otimes k}\right|_{H} \subset \infty \cdot \lambda_{H}$.

We remark that to pass from the simple to the semi-simple case, one can use the argument proving Lemma 2.2.6 of [Co2], and the fact that every positivedefinite spherical function on a semi-simple group is the product of positivedefinite spherical functions on the simple components. The latter fact follows from e.g. [He 2, Thm. 3.7, p. 414], together with the fact that an irreducible representation of $G$ is a tensor product of irreducible representations of the simple components. A useful consequence of the spectral transfer principle is given by the following:

Theorem 1. Let $G$ and $\pi$ be as above, and let $\mu \in P(G)$ be an arbitrary Borel probability measure on $G$. Then : (i) $\|\pi(\mu)\| \leq\left\|\lambda_{G}(\mu)\right\|^{1 / n(G)}$. (ii) If $\mu$ is supported on a closed unimodular subgroup $H \subset G$, then $\|\pi(\mu)\| \leq\left\|\lambda_{H}(\mu)\right\|^{1 / n(G)}$. (iii) In particular, if $G$ acts ergodically on $(X, \mathcal{B}, m)$, and $\pi_{0}$ denotes the representation of $G$ in $L_{0}^{2}(X)=\left\{f \mid \int_{X} f d m=0\right\}$, then $\left\|\pi_{0}(\mu)\right\| \leq\left\|\lambda_{G}(\mu)\right\|^{1 / n(G)}$.

Proof. For (iii), fix a real valued $f \in L_{0}^{2}(X)$, and then by Jensen's inequality, using $n=2 k$

$$
\begin{array}{r}
\|\pi(\mu) f\|^{2 n}=\left(\int_{G}\langle\pi(g) f, f\rangle d\left(\mu^{*} * \mu\right)\right)^{n} \leq \int_{G}(\langle\pi(g) f, f\rangle)^{n} d\left(\mu^{*} * \mu\right) \\
=\int_{G}\left\langle\pi^{\otimes n}(g) f^{\otimes n}, f^{\otimes n}\right\rangle d\left(\mu^{*} * \mu\right)=\left\|\pi^{\otimes n}(\mu) f^{\otimes n}\right\|^{2} \\
\leq\left\|\pi^{\otimes n}(\mu)\right\|^{2}\left\|f^{\otimes n}\right\|^{2} \leq\left\|\lambda_{G}(\mu)\right\|^{2}\|f\|^{2 n},
\end{array}
$$


and the inequality follows by taking the $2 n$-th root. For a general representation of $G$ apply the previous argument to $(\pi \otimes \bar{\pi})^{k}$, where $\bar{\pi}$ is the contregredient representation, (see e.g. [Co2, proof of Lemme 2.2.6]). The same arguments apply to $H \subset G$.

\section{Remarks.}

1) The spectral transfer principle and Theorem 1 can be viewed as an analog, in the context of semi-simple Lie groups with property $T$, of the transfer principle for amenable groups $([\mathrm{W}][\mathrm{Ca}][\mathrm{C}-\mathrm{W}][\mathrm{Hz} 1][\mathrm{Co} 3][\mathrm{Co} 4])$. In the amenable case, one bounds the operator norm of the maximal operator $M_{\mu} f=\sup _{t>1}\left|\pi\left(\mu_{t}\right) f\right|$ in an arbitrary action $\pi$, in terms of the norm of $M_{\mu}^{\mathrm{reg}} f=\sup _{t \geq 1}\left|\lambda_{G}\left(\mu_{t}\right) f\right|$ where $\lambda_{G}$ is the regular representation. Similarly, Theorem 1 gives a bound for $\left\|\pi_{0}(\mu)\right\|$ in an arbitrary ergodic action, in terms of a bound for $\|\lambda(\mu)\|$, where $\lambda$ is the regular representation. For more on this point of view see [N4][N6].

2) For amenable groups, $\left\|\lambda_{G}(\mu)\right\|=1$ for every probability measure. In contrast, typically for a family $\mu_{t}$ of probability measures on a simple non-compact Kazhdan group $G$, it is possible to obtain the estimate $\left\|\lambda_{G}\left(\mu_{t}\right)\right\| \leq C \exp (-\delta t)$ for some $\delta>0$. Therefore, in any measure preserving ergodic action of $G$ : $\left\|\pi_{0}\left(\mu_{t}\right)\right\| \leq C \exp (-t \delta / n(G))$. We will use such decay estimates to prove norm inequalities for the exponential-maximal function

$$
f_{\mu}^{*}(x)=\sup _{t \geq 1} \exp (t \delta / 2 n(G))\left|\pi\left(\mu_{t}\right) f(x)\right|,
$$

for absolutely continuous as well as singular averages, including averages supported on discrete subgroups.

3) The crucial fact in the considerations above is the existence, given an action of $G$ on $(X, B, m)$, of a finite $n \in \mathbb{N}$ such that $\pi_{0}^{\otimes n} \subset \infty \cdot \lambda_{G}$. This property holds also for many natural ergodic actions of general semi-simple groups. For example, if $\Gamma$ is a lattice in any simple non-compact Lie group $G$ then $L_{0}^{2}(G / \Gamma)$ has this property. Furthermore, simple non-compact algebraic groups over locallycompact non-discrete fields (and more generally $S$-arithmetic groups) also have many actions with this property, and in fact satisfy property $T$ if their split rank is at least two. All of the results below will apply to such actions, which we refer to as actions with a spectral gap.

4)We note that the unitary dual of many higher rank semi-simple algebraic groups is not completely classified, and even when the classification is known, it is not easy to apply. However, the spectral transfer principle and Theorem 1 allow control of the norms of the natural integral operators associated with the group action, using the information contained in the regular representation only, which is a significant simplification.

5) The spectral transfer principle and Theorem 1, can be applied not only to integral operator on $G$ but also to differential operators in the universal enveloping algebra, acting in an arbitrary representation. For further discussion of applications to spectral multipliers and an estimate of the spectral gap of certain elliptic operators we refer to [N5]. For a detailed analysis of the case of radial averages, we refer to [N1-3] [NS1-2] and [MNS]. 
1.2. Definitions and preliminaries. Throughout the following, we will consider a locally compact second countable (lcsc) group $G$ acting measurably by measure preserving transformations on a $\sigma$-finite measure space $(X, \mathcal{B}, m)$. We let $\pi$ denote the unitary representation of $G$ in $L^{2}(X)$, and let $L_{I}^{2}(X)$ denote the closed subspace of $G$-invariant vectors. $L_{0}^{2}(X)$ will denote the orthogonal complement to $L_{I}^{2}(X)$, and $\pi_{0}$ the representation of $G$ on $L_{0}^{2}(X)$. We assume that there exists an even $n=n(X)=2 k$ such that $\pi_{0}^{\otimes n} \subset \infty \cdot \lambda_{G}$ and $\left(\pi_{0} \otimes \bar{\pi}_{0}\right)^{\otimes k} \subset \infty \cdot \lambda_{G}$. By Theorem $1(\S 1.1)$, we then have $\left\|\pi_{0}(\mu)\right\| \leq\|\lambda(\mu)\|^{1 / n}$ where $\lambda$ is the regular representation of $G$ in $L^{2}(G)$, and $\mu \in P(G)$ an arbitrary Borel probability measure.

Recall that when $m(X)=1$, the $G$-action is called ergodic if the only $G$ invariant functions in $L^{2}(X)$ are the constant functions.

Given any finite Borel measure $\mu$ on $G$, consider the operator $\pi(\mu)$ defined by $\pi(\mu) f(x)=\int_{G} f\left(g^{-1} x\right) d \mu(g)$, acting on $L^{p}(X), 1 \leq p \leq \infty$.

Definition 1. Let $\nu_{t}, t \in \mathbb{R}_{+}$(or $t \in \mathbb{Z}_{+}$) be a one parameter family of probability measures on $G$, such that $t \rightarrow \nu_{t}$ is $w^{*}$-continuous in the $w^{*}$-topology on $P(G)$.

(1) Given an ergodic probability-measure-preserving action of $G$ on $(X, \mathcal{B}, m), \nu_{t}$ is called a pointwise ergodic family in $L^{p}(X)$ if for every $f \in L^{p}(X), \lim _{t \longrightarrow \infty} \pi\left(\nu_{t}\right) f(x)=\int_{X} f(x) d m$, where the convergence is pointwise almost everywhere and in the $L^{p}$ norm.

(2) $f_{\nu}^{*}(x)=\sup _{t>1}\left|\pi\left(\nu_{t}\right) f(x)\right|$ is the global part of the maximal operator.

(3) The family $\nu_{t}$ satisfies the strong $\left(L^{r}, L^{p}\right)$ exponential maximal inequality with parameter $\delta$ if

$$
\left\|\sup _{t \geqslant 1} \exp (\delta t)\left|\pi\left(\nu_{t}\right) f-\int_{X} f d m\right|\right\|_{r} \leq C_{p}\|f\|_{p}
$$

(4) The family $\nu_{t}$ converges pointwise exponentially fast to the ergodic mean, with rate $\delta$ in $L^{p}$, if for every $f \in L^{p}(X)$ the following holds for almost all $x \in X$ :

$$
\left|\pi\left(\nu_{t}\right) f(x)-\int_{X} f(x) d m\right| \leq C_{p}(x, f) e^{-\delta t} .
$$

(5) Given $\theta, \theta_{p}$ is defined by : $\theta_{p}=2 \theta\left(1-\frac{1}{p}\right)$ if $1 \leq p \leq 2$ and $\theta_{p}=\frac{2 \theta}{p}$ if $p \geq 2$. Also : $\theta_{p}^{\prime}=\theta_{p}$ if $1<p \leq 2$, and $\theta_{p}^{\prime}=\theta$ otherwise.

Definition 2. A one-parameter family $\nu_{t}, t \geq 0$ of probability measures on $G$ will be called :

(1) Roughly Monotone, if $\nu_{t} \leq B \sum_{k=-\min ([t], N)}^{N} \nu_{[t]+k}$, for $t \geq 1$ and some fixed $B$ and $N$. (Here $[t]$ is the largest integer $\leq t$ ). 
(2) Uniformly Hölder Continuous with exponent $a$, if $t \mapsto \nu_{t}$ satisfies : $\left\|\nu_{t+\epsilon}-\nu_{t}\right\|_{1} \leq C|\epsilon|^{a}$, for some $0<a \leq 1,0<\epsilon \leq 1 / 2$ and all $t \geq 1$. Here $\|\nu\|_{1}$ is the total variation norm on $P(G)$.

Finally, we recall the following definition of Kazhdan's property $T$ :

Definition 3. [Ka] (see also [HV]) An lcsc group $G$ has property $T$ of Kazhdan if there exist a compact subset $Q \subseteq G$ and $\epsilon>0$ satisfying the following: Given a strongly continuous unitary representation $(\pi, \mathcal{H})$ which does not contain $G$ invariant unit vectors, for every unit vector $v \in \mathcal{H}$ there exists $g \in Q$ with $\|\pi(g) v-v\| \geq \epsilon$. The pair $(Q, \epsilon)$ is refered to as Kazhdan constants for $G$.

\section{Maximal inequalities and ergodic theorems}

\subsection{Ergodic Theorems using spectral transfer.}

We begin with a general result regarding sequences of probability measures, as well as $w^{*}$-continuous one-parameter families. We refer for definitions of terms appearing below to $\S 1.2$.

Theorem 2. Let $(X, \mathcal{B}, m)$ be a measure preserving action of an lcsc group $G$. Assume $\pi_{0}^{\otimes n} \subset \infty \cdot \lambda_{G}$, and that $\nu_{t}, t \in \mathbb{R}_{+}$satisfies $\left\|\lambda_{G}\left(\nu_{t}\right)\right\| \leq C e^{-\theta t}$, where $\theta>0$. Then

(1) The sequence $\nu_{k}, k \in \mathbb{N}$ satisfies the strong $\left(L^{p}, L^{p}\right)$-exponential maximal inequality in $L^{p}(X), 1<p \leq \infty$, with parameter $\frac{\theta_{p}}{2 n}$, namely for $f \in$ $L_{0}^{p}(X):$

$$
\left\|\sup _{k \geq 0} e^{\frac{\theta_{p}}{2 n} k}\left|\pi_{0}\left(\nu_{k}\right) f\right|\right\|_{L_{0}^{p}(X)} \leq C_{p}\|f\|_{L_{0}^{p}(X)} .
$$

(2) If $m(X)=1$, and $G$ acts ergodically, the sequence $\pi\left(\nu_{k}\right)$ converges pointwise exponentially fast to the ergodic mean, with rate $\frac{\theta_{p}^{\prime}}{2 n}$ in $L^{p}$, $1<p \leq \infty$. Explicitly, for $f \in L^{p}(X), p>1$ :

$$
\left|\pi\left(\nu_{k}\right) f(x)-\int_{X} f d m\right| \leq C_{\theta, p}(x, f) \exp \left(-\frac{\theta_{p}^{\prime}}{2 n} k\right) .
$$

(3) If $\nu_{t}$ is a roughly monotone family, than the one-parameter family $\nu_{t}$ satisfies the strong $\left(L^{p}, L^{p}\right)$ maximal inequality in $L^{p}(X), 1<p \leq \infty$, with parameter $\delta=0$.

(4) If in addition $\nu_{t}$ is uniformly Hölder continuous (with exponent a), then it satisfies the strong $\left(L^{r}, L^{p}\right)$-exponential maximal inequality with parameter $\delta=\delta(r, p, \theta, a)>0$. Furthermore, $\nu_{t}$ converges pointwise exponentially fact to the ergodic mean, with rate $\delta$ in $L^{p}$. Explicitly, for $f \in L^{p}(X), 1<p<\infty$ :

$$
\left|\pi\left(\nu_{t}\right) f(x)-\int_{X} f(x) d m\right| \leq C(x, f) \exp (-\delta t) .
$$


Proof. (1) and (2) follow as in the proof of Theorem 6 in [MNS], which considers the radial case. (3) and (4) follow using the same argument used in the proof of Theorem 4 in [MNS]. The assumption there that the averages are radial and $(\alpha, \theta)$-admissible is used only to obtain the norm estimate $\left\|\pi\left(\nu_{t}\right)\right\| \leq C \exp (-\theta t)$. We refer to [MNS] for further details and for the explicit dependence of $\delta$ on $(r, p, \theta, a)$.

Remark. To obtain an $L^{2}$ maximal inequality and pointwise convergence for $L^{2}$ functions, for a general sequence of averages $\eta_{k}$ acting in $L^{2}(X)$, it suffices to assume only $\sum_{k=0}^{\infty}\left\|\lambda_{G}\left(\eta_{k}\right)\right\|^{2 / n(X)}<\infty$, provided that $\pi_{0}^{\otimes n(X)} \subset \infty \cdot \lambda_{G}$.

Recall now the following definition, introduced in [Co1, §7 ] (see also [KS1]) :

Definition 4. An lcsc group $G$ will be called a Kunze-Stein group if for every $1 \leq p<2$ there exists a constant $K_{p}$ satisfying : $\|F * f\|_{2} \leq K_{p}\|F\|_{p}\|f\|_{2}$, for every $F \in L^{p}(G)$ and $f \in L^{2}(G)$.

We can now consider the case of case of absolutely continuous averages :

Theorem 3. Assume $G$ is a Kunze-Stein group. Let $f_{t} \in L^{1}(G), f_{t} \geq 0$, $\int_{G} f_{t}(g) d g=1$. Assume that the family of probability measures $\nu_{t}$ with density $f_{t}$ form a roughly monotone and uniformly Hölder continuous family. Let $\left\|f_{t}\right\|_{L^{r}(G)} \leq C e^{-\theta t}$ for some $1<r<2$ and some $\theta>0$. If $\pi_{0}^{\otimes n} \subset \infty \cdot \lambda_{G}$, then $\pi\left(\nu_{t}\right)$ satisfies the conclusions of Theorem 2 .

Proof. By assumption, since $G$ is a Kunze-Stein group, the norm of the convolution operator $\lambda_{G}\left(f_{t}\right)$ acting on $L^{2}(G)$ is bounded by $K_{r}\left\|f_{t}\right\|_{L^{r}(G)}$. Hence

$$
\left\|\pi_{0}\left(\nu_{t}\right)\right\|=\left\|\pi_{0}\left(f_{t}\right)\right\| \leq\left\|\lambda_{G}\left(f_{t}\right)\right\|^{1 / n} \leq K_{r}^{1 / n}\left\|f_{t}\right\|_{L^{r}(G)}^{1 / n} \leq C(r) \exp (-\theta t / n) .
$$

Therefore the assumptions of Theorem 2 are satisfied.

More generally, the Kunze-Stein phenomenon and the spectral transfer principle combine to give the following

Theorem 4. Let $G$ be a Kunze-Stein group. Assume $\pi_{0}^{\otimes n} \subset \infty \cdot \lambda_{G}$. Let $F \in L^{1}(G) \cap L^{p}(G), 1 \leq p<\frac{2 n}{2 n-1}$. Then $\left\|\pi_{0}(F)\right\| \leq C_{p}\|F\|_{L^{p}(G)}$, i.e. $\pi_{0}$ can be extended to $L^{p}(G)$.

Proof. By [KS1, Lemma 27] (see also [Co1], Lemma 1.1), for an arbitrary unitary representation $\tau$ the following holds for the conjugate exponents $\frac{1}{p}+\frac{1}{q}=1$ :

$\langle\tau(g) v, w\rangle \in L^{q}(G)$ for all $v, w \in \mathcal{H}_{\tau}$ if and only if $\|\tau(F)\| \leq C_{p}\|F\|_{L^{p}(G)}$ for all $F \in L^{1}(G) \cap L^{p}(G)$.

It follows that for the regular representation $\lambda_{G}$ of a Kunze-Stein group $G$, we have $\langle\lambda(g) v, w\rangle \in L^{q}(G)\left(v, w \in L^{2}(G)\right)$ for all $q>2$. Equivalently $\left\|\lambda_{G}(F)\right\| \leq C_{p}\|F\|_{L^{p}(G)}$ for every $1 \leq p<2$ and $F \in L^{p}(G)$. Hence if $\pi_{0}^{\otimes n} \subset$ $\infty \cdot \lambda_{G}$ then $\left\langle\pi_{0}(g) v, w\right\rangle \in L^{q n}(G)\left(u, v \in \mathcal{H}_{\pi}\right)$ for all $q>2$, and so by the Lemma 
quoted, $\left\|\pi_{0}(F)\right\| \leq C_{p}\|F\|_{L^{p}(G)}$ for every $1 \leq p<\frac{2 n}{2 n-1}\left(\right.$ since $\left.\frac{1}{2 n}+\frac{2 n-1}{2 n}=1\right)$, and every $F \in L^{1}(G) \cap L^{p}(G)$.

We also note the following result, which establishes a general maximal inequality for averages on the group, valid when the space has infinite $\sigma$-finite invariant measure. A large class of examples is provided by transitive actions of the group on its homogeneous spaces carrying an infinite $G$-invariant measure. Note that the maximal inequality requires no covering arguments and no volume or growth properties, and is based solely on the spectral assumption.

Theorem 5. Assume $G$ is a Kunze-Stein group. Let $m(X)=\infty$, and assume that $L_{I}(X)=0$ and $\pi^{\otimes n} \subset \infty \cdot \lambda_{G}$. Let $A_{t}, t>0$ be an incresing family of sets of positive finite measure satisfying $\cup_{t>0} A_{t}=G$, such that $t(g)=\inf \left\{t \mid g \in A_{t}\right\}$ is measurable. Let $\nu_{t}$ be the probability measures on $G$ with density $\chi_{A_{t}} / \operatorname{vol} A_{t}$, and assume $t \mapsto \nu_{t}$ is $w^{*}$-continuous. Then

(1) $\left\|f_{\nu}^{*}\right\|_{L^{2}(X)}=\left\|\sup _{t>1} \mid \pi\left(\nu_{t}\right) f\right\|_{L^{2}(X)} \leq C\|f\|_{L^{2}(X)}$.

(2) For every $p \geq 2:\left\|f_{\nu}^{*}\right\|_{L^{p}(X)} \leq C_{p}\|f\|_{L^{p}(X)}$.

(3) If $\eta_{k}$ is any sequence of probability measures satifying $\left\|\lambda_{G}\left(\eta_{k}\right)\right\| \leq$ $C \exp (-\theta k)$, for some $\theta>0$, then $\sum_{k=0}^{\infty} \exp \left(k p \theta_{p} / 2 n\right)\left|\pi\left(\eta_{k}\right) f(x)\right|^{p}<\infty$ almost everywhere, and $\eta_{k}$ satisfies the strong $\left(L^{p}, L^{p}\right)$ exponential maximal inequality in $L^{p}$ with parameter $\theta_{p} / 2 n, 1<p \leq \infty$.

(4) In particular, the last property is satisfied by the spherical averages $\sigma_{k}^{H}=$ $m_{K} * \delta_{\exp (k H)} * m_{K}$, on connected semi-simple Lie groups with finite center and no compact factors, for every $H \in \mathfrak{a}, H \neq 0$ (see $\S 2.2$ for the notation).

Proof. (1) We use the argument of [Cl-St, Lemma 2]. The function $t(g)=$ $\inf \left\{t \mid g \in A_{t}\right\}$ is measurable, and we define $V(g)=\left(1+\operatorname{vol} A_{t(g)}\right)^{-1}$. Then $\frac{1}{\operatorname{vol} A_{t}} \chi_{A_{t}}(g) \leq\left(1+1 / \operatorname{vol}\left(A_{1}\right)\right) V(g)$ for every $g \in G$, if $t \geq 1$. Hence for every $f \in L^{2}(X)$,

$$
f_{\nu}^{*}(x) \leq C \pi(V)|f(x)| \text { and hence }\left\|f_{\nu}^{*}\right\|_{L^{2}(X)} \leq C\|\pi(V)\|\|f\|_{L^{2}(X)} .
$$

The distribution of $V(g)$ is estimated by : $m_{G}(\{\mid V(g)>s\}) \leq\left(1+\operatorname{vol}\left(A_{1}\right)^{-1}\right) / s$ for $s>0$ and therefore $V \in L^{p}(G)$ for every $p>1$. By Theorem 4 , it follows that $\pi(V)$ is a bounded operator on $L^{2}(X)$, so that $\left\|f_{\nu}^{*}\right\|_{L^{2}(X)} \leq C\|f\|_{L^{2}(X)}$.

(2) The subadditive operator $f \mapsto f_{\nu}^{*}$ satisfies the strong $(2,2)$ and $(\infty, \infty)$ maximal inequalities, and hence also the strong $(p, p)$ maximal inequality for $2 \leq p \leq \infty$, by the Riesz interpolation theorem.

(3) This fact is established as in Theorem 2, part (1).

(4) The fact that the spherical averages above satisfy the norm estimate of part (3) follows from spherical functions estimates that can be found e.g. in $[\mathrm{CHH}][\mathrm{KaSp}][\mathrm{KM}]$ or $[\mathrm{MNS}]$.

Remark. It is unknown whether a (weak or strong) maximal inequality holds in $L^{p}, 1 \leq p<2$ for general $\nu_{t}$. We remark that a strong maximal inequality 
does hold for $1<p \leq \infty$ for many bi- $K$-invariant averages and refer to [MNS] for a proof.

\subsection{Estimating convolution norms on semi-simple groups.}

Consider now the case of a connected semi-simple Lie group $G$ with finite center and no compact factors. Here the majorization principle due to C. Herz $[\mathrm{Hz}]$ has a useful corollary due to M. Cowling, U. Haagerup and R. Howe [CHH], which allows us to estimate the norm of the convolution operator $\lambda_{G}(f)$ on $L^{2}(G)$, as follows :

$$
\left\|\lambda_{G}(f)\right\| \leq \int_{G}\left(\int_{K} \int_{K}\left|f\left(k g k^{\prime}\right)\right|^{2} d k d k^{\prime}\right)^{1 / 2} \Xi(g) d g,
$$

where $\Xi(g)$ is the Harish Chandra $\Xi$ function, given by:

$$
\Xi(g)=\int_{K} \Delta^{-1 / 2}(g k) d k=\left\langle\rho_{0}(g) 1,1\right\rangle .
$$

Here $\rho_{0}$ is the representation of $G$ induced from the trivial representation of $P$, the minimal parabolic subgroup occurring in the Iwasawa decomposition $G=K P$, and $\Delta$ is the modular function of $P$ ( $\Delta$ is extended to a left $K-$ invariant function on $G$ ). The expression of Haar measure in terms of the Iwasawa decomposition is $\int_{G} f(g) d g=\int_{K} \int_{P} f(k p) \Delta(p) d p d k, d p$ left Haar measure on $P, \Delta(p) d p$ right Haar measure on $P$.

We recall that the $\Xi$-function satisfies the basic estimate (see e.g. $[\mathrm{G}-\mathrm{V}, \mathrm{p}$. 161]) :

$$
\Xi\left(k \exp (t H) k^{\prime}\right) \leq C(1+\|H\|)^{d} e^{-t \rho(H)}
$$

where $\rho=$ half the sum of positive roots of $\operatorname{ad}(\mathfrak{a})$ in $\mathfrak{g}$, and $C, d$ depend on $G$ only.

The estimate above for $\lambda_{G}(f)$ gives a simple and explicit integral criterion for a family $f_{t} \in L^{1}(G), t \in \mathbb{R}_{+}$to satisfy strong exponential-maximal inequality in every $L^{p}(X), 1<p \leq \infty$. In particular it allows us to estimate the convolution norm of a non-radial function in terms of an associated radial function, which is much easier to control.

As an example, we consider the following family of non-radial averages, defined using the Iwasawa coordinates $G=\mathrm{KAN}$. As is well known (e.g. [He2], p. 181) Haar measure can be normalized so that in horospherical coordinates it is given by

$$
\int_{G} f(g) d g=\int_{K \times \mathfrak{a} \times N} f\left(k e^{H} n\right) e^{2 \rho(H)} d k d H d n .
$$

Now let $h_{t}$ denote the probability measure on $G$ whose density is given by $\chi_{U_{t}} / m_{G}\left(U_{t}\right)$, where $U_{t}=\left\{k e^{H} n \mid k \in K,\|H\| \leq t, n \in N_{0}\right\}$. Here $N_{0}$ is a fixed compact neighborhood of the identity in $N$. We remark that pointwise ergodic theorems for certain averages defined using Iwasawa coordinates were proved by A. Templeman [T]. We will prove the following : 
Theorem 6. Let $G$ be a connected semi-simple Lie group with finite-center and no compact factors. Then $\left\|\lambda_{G}\left(h_{t}\right)\right\| \leq C \exp (-\theta t), \theta>0, h_{t}$ is roughly monotone and uniformly Hölder continuous, and hence $h_{t}$ satisfies the conclusions of Theorem 2, provided $\pi_{0}^{\otimes n} \subset \infty \cdot \lambda_{G}$.

Proof. We start by estimating $\lambda_{G}\left(h_{t}\right)$, using the majorization formula above. Let us first estimate the translation length in $G / K$ of elements $g$ in $U_{t}$ :

$$
\begin{aligned}
& d\left(k e^{H} n K, K\right) \leq d\left(e^{H} n K, e^{H} K\right)+d\left(e^{H} K, K\right) \\
= & d(n K, K)+d\left(e^{H} K, K\right) \leq \max _{n \in N_{0}} d(n K, K)+d\left(e^{H} K, K\right)=d_{0}+\|H\| \leq d_{0}+t .
\end{aligned}
$$

Hence the set $U_{t} K=K U_{t} K$ in $G / K$ is contained in a ball $B_{t+d_{0}}(o) \subset G / K$ of radius $d_{0}+t$ and center $o=[K]$. Let $\mathfrak{a}_{r}$ denote the ball in $\mathfrak{a}$ with radius $r$ centered at the origin. Integrate $\Xi(g)$, which is bi- $K$-invariant on $B_{r}^{\prime}=\{g \mid d(g K, K) \leq r\}$, using Haar measure in polar coordinates (see e.g. [G-V, p. 73]). Then, letting $m_{\alpha}$ denote the multiplicity of the root $\alpha$ :

$$
\begin{aligned}
& \int_{B_{r}^{\prime}} \Xi(g) d g=|W| \int_{\mathfrak{a}_{r} \cap \mathfrak{a}_{+}} \Xi\left(e^{H}\right) \prod_{\alpha \in \Sigma^{+}} s h(\alpha(H))^{m_{\alpha}} d H \\
& \leq C \int_{\mathfrak{a}_{r} \cap \mathfrak{a}_{+}} \Xi\left(e^{H}\right) \exp (2 \rho(H)) d H .
\end{aligned}
$$

By definition of $U_{t}$, we have $m_{G}\left(U_{t}\right)=\int_{\mathfrak{a}_{t}} \exp (2 \rho(H)) d H \cdot m_{N}\left(N_{0}\right)$, where $m_{N}$ denote Haar measure on $N$. Now, using the estimate for the translation length, we obtain :

$$
\int_{K} \int_{K}\left|\chi_{U_{t}}\left(k g k^{\prime}\right)\right|^{2} d k d k^{\prime} \leq \chi_{B_{t+d_{0}}^{\prime}}(g)
$$

Hence:

$$
\begin{gathered}
\| \lambda_{G}\left(h_{t} \| \leq \int_{G}\left(\int_{K} \int_{K}\left|m_{G}\left(U_{t}\right)^{-1} \chi_{U_{t}}\left(k g k^{\prime}\right)\right|^{2} d k d k^{\prime}\right)^{1 / 2} \Xi(g) d g \leq\right. \\
\leq \frac{1}{m_{G}\left(U_{t}\right)} \int_{B_{t+d_{0}}^{\prime}} \Xi(g) d g \leq \frac{C}{m_{G}\left(U_{t}\right)} \int_{\mathfrak{a}_{t+d_{0}} \cap \mathfrak{a}_{+}} \Xi\left(e^{H}\right) e^{2 \rho(H)} d H \leq \\
\leq \frac{C^{\prime} \int_{\mathfrak{a}_{t+d_{0}} \cap \mathfrak{a}_{+}} e^{-\rho(H)} e^{2 \rho(H)}(1+\|H\|)^{d} d H}{m_{N}\left(N_{0}\right) \int_{\mathfrak{a}_{t} \cap \mathfrak{a}_{+}} e^{2 \rho(H)} d H} \leq B e^{-\theta t} .
\end{gathered}
$$

Here $\theta=\theta\left(G, d_{0}\right)>0$. Furthermore, it is evident that $h_{t}$ is roughly monotone, and in fact $h_{t} \leq C h_{[t]+1}$ for a fixed $C$, using the explicit form of the density of $h_{t}$. Also, $\left\|h_{t+\epsilon}-h_{t}\right\|_{L^{1}(G)} \leq C|\epsilon|$ for all $t \geq 1$, if $0<\epsilon \leq 1 / 2$, again by a direct computation using the density (see [MNS, §3] for a similar computation for the ball averages). Hence the family $h_{t}$ is uniformly Hölder continuous, and so $h_{t}$ satisfies the assumptions of Theorem 2 with the parameter $\theta>0$, and the proof is complete. 
Fix now $H_{0} \in \mathfrak{a}_{+}$satisfying $\left\|H_{0}\right\|=1$, and consider the sets $V_{t}=\left\{k e^{H} n \mid\right.$ $\left.k \in K,\left\|H-t H_{0}\right\| \leq 1, n \in N_{0}\right\}$, and the averages $v_{t}=\chi_{V_{t} / m_{G}\left(V_{t}\right)}$. These averages are an absolutely continuous version of the singular averages $w_{t}^{H}=$ $m_{K} * \delta_{\exp t H_{0}}$, introduced by W. Veech [V], which were shown in [V] to satisfy a mean ergodic theorem in $L^{p}(X), 1 \leq p<\infty$. We have :

Theorem 7. Let $G$ be a simple non-compact Lie group with finite center and real rank one. Then the family $v_{t}$ is a roughly monotone family which is uniformly Hölder continuous, and satisfies $\left\|\lambda_{G}\left(v_{t}\right)\right\| \leq C \exp (-\theta t), \theta>0$.In any action satisfying $\pi_{0}^{\otimes n} \subset \infty \cdot \lambda_{G}$, the family $v_{t}$ satisfies the conclusions of Theorem 2.

Proof. The estimate $\left\|\lambda_{G}\left(v_{t}\right)\right\| \leq C \exp (-\theta t), \theta>0$ follows by a similar computation, and again $v_{t}$ is a roughly monotone family which is uniformly Hölder continuous by a direct computation involving the density.

\section{Applying the spectral transfer principle : Singular averages}

\subsection{Averages supported on closed unimodular subgroups.}

In this section we consider averages $\mu_{t}$ supported on a closed subgroup $H \subset$ $G$, which are of course all singular with respect to Haar measure on $G$. By Theorem 1, the problem of estimating the operator norm $\pi_{0}\left(\mu_{t}\right)$ is reduced to estimating the norm of $\lambda_{H}\left(\mu_{t}\right)$ as a convolution operator on $L^{2}(H)$. Using Theorem 7, we conclude the following about the behaviour of averages on realrank-one subgroups (which do not have propert $T$, in general) :

Corollary 3.1. Let $G$ be a connected semi-simple Lie group with finite center and no compact factors, $L \subset G$ a connected almost simple subgroup of real rank one. If $(X, \mathcal{B}, m)$ is a $G$-space satisfying $\pi_{0}^{\otimes n} \subset \infty \cdot \lambda_{G}$, then the horospherical averages $h_{t}^{L}$ and $v_{t}^{L}$ satisfy the conclusions of Theorem 2 . The same applies also to the family $\beta_{t}^{L}$ of ball averages on $L$.

\subsection{Averages supported on discrete hyperbolic subgroups.}

Fix now a discrete subgroup $\Gamma \subset G$ with $S=S^{-1}$ a finite symmetric set of generators for $\Gamma$. Consider the associated word-length and define the following averages (see e.g. [N3][N4]) :

\section{Definition 4.}

(1) $\sigma_{n}=\frac{1}{\# S_{n}} \sum_{w \in S_{n}} w$, where $S_{n}=\{w:|w|=n\}$ is the sphere of radius $n$, with center $e$.

(2) $\beta_{n}=\frac{1}{\# B_{n}} \sum_{w \in B_{n}} w$, where $B_{n}=\{w:|w| \leq n\}$ denotes the ball of radius $n$ with center $e$.

Recall now the following convolution theorem due to P. Jolissaint $[\mathrm{J}]$ :

Theorem 3.2. [J] Let $\Gamma$ be a word-hyperbolic (non-elementary) group, $S=$ $S^{-1}$ a finite symmetric set of generators, and $L(g)=|g|$ the associated length 
function. Then there exist two positive constants $s=s(\Gamma, S)$ and $C=C(\Gamma, S)$, such that for any finitely supported function $f \in \ell^{1}(\Gamma)$ :

$$
\|\lambda(f)\| \leq C\left\|f \cdot(1+L)^{s}\right\|_{\ell^{2}(\Gamma)} .
$$

This result implies the following

Corollary 3.3. Let $\Gamma$ denote a non-elementary word-hyperbolic group, $S=S^{-1}$ a finite symmetric set of generators. Let $q_{\sigma}=q_{\sigma}(\Gamma, S)=\lim _{n \rightarrow \infty} \frac{1}{n} \log \left|S_{n}\right|$ (see [Coo]). Then:

(1) $\left\|\lambda\left(\sigma_{k}\right)\right\| \leq B q^{-k}$, and $\left\|\lambda\left(\beta_{k}\right)\right\| \leq B q^{-k}$, for any $1<q<\sqrt{q_{\sigma}}$ and some $B=B_{q}>0$.

(2) $\sigma_{k}$ and $\beta_{k}$ satisfy the strong $\left(\ell^{p}, \ell^{p}\right)$ exponential maximal inequality in $\ell^{p}(\Gamma)$, with parameter $\theta_{p}$, where $\theta=\theta_{2}=\frac{1}{4} \log q_{\sigma}$.

Proof. It is proved in [Coo] that there exist $q_{\sigma}=q_{\sigma}(\Gamma, S), C_{1}>0$ depending only on $(\Gamma, S)$, satisfying: $C_{1}^{-1} q_{\sigma}^{k} \leq\left|S_{k}\right| \leq C_{1} q_{\sigma}^{k}$. Hence

$$
\begin{aligned}
\left\|(1+L)^{s} \cdot \sigma_{k}\right\|_{\ell^{2}(\Gamma)} & =\left[\sum_{w \in S_{k}}\left(\frac{1}{\left|S_{k}\right|}\right)^{2}(1+k)^{2 s}\right]^{1 / 2} \\
& =\left|S_{k}\right|^{-1}\left(\left|S_{k}\right|(k+1)^{2 s}\right)^{1 / 2} \leq\left(C_{1}^{-1} q_{\sigma}^{k}\right)^{-1 / 2}(k+1)^{s} \leq B q^{-k}
\end{aligned}
$$

provided $B \geq \sqrt{C_{1}}, 1<q<\sqrt{q_{\sigma}}$. The result follows from the convolution theorem 3.2.

For the ball averages, we write $\left|B_{k}\right| \beta_{k}=\sum_{j=1}^{k}\left|S_{j}\right| \sigma_{j}$, so that

$$
\beta_{k}=\sum_{j=0}^{k} \frac{\left|S_{j}\right|}{\left|B_{k}\right|} \sigma_{j} \leq C_{2} \sum_{j=0}^{k} q_{\sigma}^{j-k} \sigma_{j}
$$

Hence, using the estimate for the spectral norm of the spheres $\sigma_{k}$, the result for $\beta_{k}$ follows.

The exponential-maximal inequality in $\ell^{2}(\Gamma)$ with parameter $\theta<\frac{1}{2} \log q_{\sigma}$ is an immediate consequence of the norm estimate, and for $\ell^{p}(\Gamma), 1<p \leq \infty$ follows by Riesz-Thorin interpolation.

The previous result estimates the spectral norm $\left\|\lambda_{\Gamma}(\mu)\right\|$ of operators $\mu \in$ $\ell^{1}(\Gamma)$ acting by convolutions in the regular representation. When $\Gamma \subset G$ is discrete, the spectral transfer principle implies $\left(\left.\pi_{0}\right|_{\Gamma}\right)^{\otimes n} \subset \infty \cdot \lambda_{\Gamma}$. We remark that this fact was applied already by G. A. Margulis in $[\mathrm{M} 1, \S 3]$ in the case $\Gamma$ is a free non-Abelian subgroup. By Theorem $1,\left\|\pi_{0}(\mu)\right\| \leq\left\|\lambda_{\Gamma}(\mu)\right\|^{1 / n}$ for any $\mu \in P(\Gamma)$. Hence the following conclusion (which improves some results in $[\mathrm{F}-\mathrm{N}]$, for actions possesing a spectral gap). 
Theorem 8. Let $G$ be an lcsc group, and let $\Gamma \subset G$ be a non-elementary wordhyperbolic discrete subgroup of $G$ with a fixed finite symmetric set of generators $S$. Assume $G$ acts on a probability space $X$ and $\pi_{0}^{\otimes n} \subset \infty \cdot \lambda_{G}$. Then:

(1) $\sigma_{k}$ satisfies the strong $\left(L^{p}, L^{p}\right)$ exponential-maximal inequality, $1<p \leq$ $\infty$, with parameter $\theta_{p}$, where $\theta=\frac{1}{4 n} \log q_{\sigma}$.

(2) The pointwise convergence to the ergodic mean in $L^{p}$ is exponentially fast, with rate $\theta_{p}^{\prime}$, where $\theta=\frac{1}{4 n} \log q_{\sigma}$.

(3) Both inequalities above are valid when $\sigma_{k}$ is replaced by $\beta_{k}$.

\section{Examples 3.4.}

(1) Let $\Gamma=\mathbb{F}_{r}$, the free non-abelian group on $r$ generators, and assume $S=S^{-1}$ is a free set of generators. Then $\left\|\lambda\left(\sigma_{k}\right)\right\|=\varphi_{0}\left(\sigma_{k}\right)$, where $\varphi_{0}$ (the Harish-Chandra $\Xi$-function for this case) satisfies (see e.g. [N3, NS1] $):\left|\varphi_{0}\left(\sigma_{k}\right)\right|=\left(1+C_{r} k\right)(2 r-1)^{-k / 2} \leq B q^{-k}$, provided $q<\sqrt{2 r-1}$. By Theorem 8, when $\Gamma$ is embedded as a discrete subgroup of a group $G$, for every representation $\pi_{0}$ of $G$ satisfying $\pi_{0}^{\otimes n} \subset \infty \cdot \lambda_{G}$, we have $\left\|\pi_{0}\left(\sigma_{k}\right)\right\| \leq B_{r} q^{-k / n}$, if $q<\sqrt{2 r-1}$.

(2) Theorem 8 applies to co-compact lattices $\Gamma$ in the simple Kazhdan groups $G=S p(n, 1), n \geq 2$, which are word-hyperbolic groups. In every ergodic action of $G$, the sphere averages on $\Gamma$ converge pointwise exponentially fast to the ergodic mean.

(3) Similar conclusions hold for most one-relator discrete subgroups $\Gamma$ of $G$, by the spectral norm estimates recently established in [CVJ, Prop. 5.4]. These imply that $\lambda_{\Gamma}\left(\eta_{n}\right) \leq C \exp (-\theta n), \theta>0$, for certain sequences $\eta_{n}$. Such estimates apply, in particular, to the fundamental group $\pi_{1}\left(M_{g}\right)$ of a closed surface of genus $g \geq 2$.

\subsection{Ergodic theorems for averages supported on lattice points.}

Let $G$ be an lcsc group, and $\Gamma \subset G$ a discrete lattice subgroup. Given a sequence of compact sets $U_{n} \subset G$ satisfying $m_{G}\left(U_{n}\right) \rightarrow \infty$, consider the sets $A_{n}=U_{n} \cap \Gamma$ of lattice points, (which are assumed non-empty) and define the averages $\alpha_{n} \in \ell^{1}(\Gamma)$ by : $\alpha_{n}=\left|A_{n}\right|^{-1} \sum_{\gamma \in A_{n}} \gamma$. For example, take $U_{n}$ as the bi- $K$-invariant balls $B_{n}=\{g \mid d(g K, K) \leq n\}$ on a connected semi-simple Lie group with finite center.

It is a natural question whether the norm of the discrete convolution operator $\lambda_{G}\left(\alpha_{n}\right)$ on $L^{2}(G)$ can be estimated by the convolution norm of the corresponding absolutely continuous averaging operator $u_{n}=\frac{1}{m_{G}\left(U_{n}\right)} \chi_{U_{n}}$. A partial answer to this question is provided by a norm estimate, stated in [B-S, §7] and due to $\mathrm{M}$. Cowling and T. Steger (see [C-S]), given as follows. Let $F$ and $\psi$ be continuous compactly supported functions on $G$, so that $\tilde{\psi}(x)=\tilde{\psi}(x \Gamma)=\sum_{\gamma \in \Gamma} \psi(x \gamma)$ satisfies $\tilde{\psi} \in L^{2}(G / \Gamma)$. Let $\pi$ be any unitary representation of $G$, and $\lambda_{G / \Gamma}$ the representation of $G$ in $L^{2}(G / \Gamma)$. 
Theorem 3.5. ([B-S], $[\mathbf{C - S}])$ Let $G$ be an lcsc group, $\Gamma$ a discrete lattice subgroup, and $F, \psi$ as above. Then:

$$
\left\|\left.\pi\right|_{\Gamma}\left(\left.\left(\psi^{*} * F * \psi\right)\right|_{\Gamma}\right)\right\| \leq\|\tilde{\psi}\|_{L^{2}(G / \Gamma)}^{2}\left\|\left(\lambda_{G / \Gamma} \otimes \pi\right)(F)\right\| .
$$

Taking $\pi=\lambda_{G}$, we have of course $\lambda_{G / \Gamma} \otimes \lambda_{G} \cong \infty \cdot \lambda_{G}$, and $\left.\lambda_{G}\right|_{\Gamma} \cong \infty \cdot \lambda_{\Gamma}$. Hence if $f_{k}=\left.\left(\psi^{*} * F_{k} * \psi\right)\right|_{\Gamma}$ is the restriction to $\Gamma$ of a sequence $F_{k} \in C_{c}(G)$, we conclude

Corollary 3.6. Let $G, \Gamma$ be as in Theorem 3.5, $F_{k}$ and $f_{k}$ as above. Then

(1) $\left\|\lambda_{\Gamma}\left(f_{k}\right)\right\| \leq C\left\|\lambda_{G}\left(F_{k}\right)\right\|$.

(2) If $\left\|\lambda_{G}\left(F_{k}\right)\right\| \leq C e^{-\delta k}$ for $F_{k} \in C_{c}(G)$, and $\delta>0$, then $f_{k} \in \ell^{1}(\Gamma)$ satisfy, in every unitary representation $\pi$ of $\Gamma$ such that $\pi^{\otimes n} \subset \infty \cdot \lambda_{\Gamma}$, the estimate: $\left\|\pi\left(f_{k}\right)\right\| \leq C e^{-\delta k / n}$.

(3) In particular, if $\pi$ arises from a measure preserving action, $\pi\left(f_{k}\right)$ satisfies a strong $\left(L^{p}, L^{p}\right)$ exponential maximal inequality with parameter $\theta_{p}, 1<$ $p \leq \infty$, where $\theta=\delta / 2 n$, and $\pi\left(f_{k}\right)$ converges exponentially fast to the ergodic mean with rate $\theta_{p}^{\prime}$.

Consider now two arbitrary probability measures $\mu_{1}$ and $\mu_{2}$ on a locally compact group $G$.

Proposition 3.7. If $\mu_{1} \leq C \mu_{2}$, and $\pi^{\otimes n} \subset \infty \cdot \lambda_{G}$, then $\left\|\pi\left(\mu_{1}\right)\right\| \leq\left\|C \lambda_{G}\left(\mu_{2}\right)\right\|^{1 / n}$. Proof. Since $\mu_{1} \leq C \mu_{2}$, we have $\left\|\lambda_{G}\left(\mu_{1}\right)\right\| \leq C\left\|\lambda_{G}\left(\mu_{2}\right)\right\|$, since $\left\|\mu_{1} * f\right\|_{L^{2}(G)} \leq$ $C\left\|\mu_{2} *|f|\right\|_{L^{2}(G)}$. Hence $\left\|\pi\left(\mu_{1}\right)\right\| \leq\left\|\lambda_{G}\left(\mu_{1}\right)\right\|^{1 / n} \leq C^{1 / n}\left\|\lambda_{G}\left(\mu_{2}\right)\right\|^{1 / n}$.

The foregoing proposition implies the following : let us say that $\mu_{1}$ is a bounded perturbation of $\mu_{2}$ with constant $C$ if $\mu_{1} \leq C \mu_{2}$.

Corollary 3.8. Let $\nu_{k}$ be any sequence of probability measures on $G$ that satisfies the estimate $\left\|\lambda_{G}\left(\nu_{k}\right)\right\| \leq B \exp (-\delta k), \delta>0$. Then any sequence of probability measures $\tilde{\nu}_{k}$ where $\tilde{\nu_{k}}$ is bounded perturbations of $\nu_{k}$ with fixed constant $C$, satisfies $\left\|\pi\left(\tilde{\nu}_{k}\right)\right\| \leq B C^{1 / n} \exp (-\delta / n k)$, provided $\pi^{\otimes n} \subset \infty \cdot \lambda_{G}$.

For example, fix any sequence of probability measures, not necessarily bi- $K$ invariant, which form a bounded perturbation of the sequence of ball averages $\beta_{n}$ on a semi-simple Kazhdan group without compact factors. Then their convolution norms satisfy an exponential decay estimate (with parameter $\theta$, say) by Corollary 3.8. Hence in any ergodic measure preserving action the averages converge pointwise exponentially fast to the ergodic mean. Furthermore the sequence of averages satisfies the strong $\left(L^{p}, L^{p}\right)$ exponential-maximal inequality with parameter $\theta_{p}, 1<p \leq \infty$, in every action, in particular for the action of $G$ by convolutions on $L^{2}(G)$.

Similarly, any sequnce of measures on $\Gamma \subset G$ which is a bounded perturbation of the measures $f_{k}$ of Corollary 3.6 satisfies an exponential decay estimate in any unitary representation $\pi$ of $\Gamma$ satisfying $\pi^{\otimes n} \subset \infty \cdot \lambda_{\Gamma}$.

Consider now $\beta_{k}=\frac{1}{v o l B_{k}} \chi_{B_{k}}(g)$ (where $B_{k}$ is the bi- $K$-invariant ball of radius $k$ in $G)$, let $B_{k}(\Gamma)=B_{k} \cap \Gamma$, and let $b_{k}=\frac{1}{\left|B_{k}(\Gamma)\right|} \sum_{\gamma \in B_{k}(\Gamma)} \gamma$. 
Theorem 9. Let $\Gamma \subset G$ be any lattice in a semisimple Lie group with finite center and without compact factors. Then the sequence $b_{k} \in \ell^{1}(\Gamma)$ satisfies:

(1) $\left\|\lambda_{\Gamma}\left(b_{k}\right)\right\| \leq C \exp -\theta k$. Here $\theta<\theta_{\beta}(G)=\lim _{t \rightarrow \infty}-\frac{1}{t} \log \left\|\lambda_{G}\left(\beta_{t}\right)\right\|$.

(2) $b_{k}$ satisfies the strong $\left(l^{p}, l^{p}\right)$ exponential-maximal inequality in $\ell^{p}(\Gamma)$, $1<p \leq \infty$, with parameter $\theta_{p}$.

(3) In any action satisfying $\pi_{0}^{\otimes n} \subset \infty \cdot \lambda_{\Gamma}, b_{k}$ satisfies the ergodic theorem stated in Corollary 3.6(3).

Proof. Let $F_{k}=\psi * \beta_{k} * \psi$, where $\psi=\beta_{1 / 2} * \beta_{1 / 2}$. $F_{k}$ is a continuous function, and its restriction $f_{k}=\left.F_{k}\right|_{\Gamma}$ satisfies $b_{k-2} \leq C f_{k}$. This follows from the fact that the number of lattice points in a ball of radius $t$ divided by the volume of the ball of radius $t$ converges to 1 , (see e.g. [E-M]). Hence the proposition follows from Corollary 3.6, Corollary 3.8, and Riesz-Thorin interpolation.

Let now $\Gamma$ be a lattice in a locally compact group $G$, and consider unitary induction of representations from $\Gamma$ to $G$ (see e.g. [HV, Ch. 3]). Let $D \subset G$ be a left fundamental domain, so $D$ maps bijectively to $G / \Gamma$ under the canonical map, and $s: d \Gamma \mapsto d \in D$ is a measurable section. We normalize the $G$-invariant measure on $G / \Gamma$ to have total mass one. Writing $g d=d^{\prime} \gamma$, the map $\alpha(g, d)=\gamma$ is a cocycle $\alpha: G \times G / \Gamma \rightarrow \Gamma$. Given any probability measure $\mu$ on $G$, we can define a probability measure $\tilde{\mu}$ on $\Gamma$, as follows. The weight at a given element $\gamma$ is given by the $\mu \times m_{G / \Gamma^{-}}$measure of the set $\left\{(g, d) \in F \times G / \Gamma \mid \alpha\left(g^{-1}, y \Gamma\right)^{-1}=\right.$ $\gamma$ \}. In other words, $\tilde{\mu}$ is the distribution function of $\alpha\left(g^{-1}, y \Gamma\right)^{-1}$, as a map defined on $\left(G \times G / \Gamma, \mu \times m_{G / \Gamma}\right)$. Let now $\tau$ be a unitary representation of $\Gamma$, and consider the representation $\pi_{\tau}$ induced to $G$. The representation space is given by $\left\{f: G / \Gamma \rightarrow \mathcal{H}_{\tau} \mid \int_{G / \Gamma}\|f(y \Gamma)\|^{2} d m_{G / \Gamma}<\infty\right\}$, and the $G$-action by $\pi_{\tau}\left(g^{-1}\right) f(y \Gamma)=\tau\left(\alpha(g, y \Gamma)^{-1} f(g y \Gamma)\right.$. It follows that if $v \in \mathcal{H}_{\tau}$, and $f_{v}: G / \Gamma \rightarrow$ $\mathcal{H}_{\tau}$ is the constant function $f_{v}(y \Gamma)=v$, then $\pi_{\tau}(\mu) f_{v}=\tau(\tilde{\mu}) v$. Therefore, we conclude :

Proposition 3.9. Let $G$ be a semi-simple Kazhdan Lie group with finite center and no compact factors, and let $\Gamma$ be a lattice in $G$. Let $\mu_{k}$ denote a sequence of probability measures on $G$, satisfying $\left\|\lambda_{G}\left(\mu_{k}\right)\right\| \leq C \exp (-\theta k)$. Let $\tilde{\mu}_{k}$ the corresponding probability measures on $\Gamma$ obtained as above by some fixed choice of a fundamental domain. If $\tau$ is any unitary representation of $\Gamma$ with no invariant unit vectors, then

$$
\left\|\tau\left(\tilde{\mu}_{k}\right)\right\| \leq\left\|\pi_{\tau}\left(\mu_{k}\right)\right\| \leq\left\|\lambda_{G}\left(\mu_{k}\right)\right\|^{1 / n(G)} \leq C^{1 / n} \exp (-\theta k / n(G)) .
$$

Proof. The estimate follows immediately from the preceding discussion and Theorem 1, together with the fact that if $\tau$ has no $\Gamma$-invariant unit vectors, then $\pi_{\tau}$ has no $G$-invariant unit vectors.

Remark. It is natural to expect that the probability measures $b_{k} \in \ell^{1}(\Gamma)$ defined above satisfy, when $\Gamma$ is a Kazhdan lattice, the estimate : $\left\|\tau\left(b_{k}\right)\right\| \leq C \exp (-k \theta)$, where $\tau$ is an arbitrary unitary representation of $\Gamma$ without invariant unit vectors, and $\theta>0$ depends only on $\Gamma$. This problem, however, remains unresolved. 


\section{Best possible pointwise ergodic theorems and maximal inequalities}

\subsection{Best possible estimates of operator norms.}

Clearly, the best possible estimate for the operator norm of a probability measure $\mu$ supported on a closed unimodular subgroup $H \subset G$, is obtained in Theorem 1 when $n=1$, and then $\left\|\pi_{0}(\mu)\right\| \leq\left\|\lambda_{H}(\mu)\right\|$.

A basic fact in the representation theory of semi-simple groups, due to D. A. Kazhdan [Ka, Lemma 2] and used repeatedly since is that certain subgroups $H$ have the following property: The restriction of any unitary representation $\pi_{0}$ of $G$ without invariant unit vectors to $H$ is weakly contained in $\infty \cdot \lambda_{H}$. As is well known (see [Ey]), a representation $\pi_{0}$ is weakly contained in $\lambda_{H}$ if and only if $\left\|\pi\left(\mu_{0}\right)\right\| \leq\left\|\lambda_{H}(\mu)\right\|$ for every (absolutely continuous) probability measure $\mu$ on $H$.

For example, subgroups $H \subset G$ of the form $H=S L_{2}(\mathbb{R}) \ltimes_{\tau} \mathbb{R}^{n}$, where $\tau$ is a representation of $S L_{2}(\mathbb{R})$ on $\mathbb{R}^{n}$ without invariant unit vectors, and $\mathbb{R}^{n}$ is a unipotent subgroup, have this restriction property, and for a proof we refer e.g. to [Co2. Thm. 2.3.1][Z, Thm. 7.3.9] and also [M, Ch.3, Prop. 4.5], where a general criterion for other examples of this type can be found.

A particularly elegant, elementary, and quantitative argument establishing this fact for the case of the defining representation $\tau$ is due to $\mathrm{R}$. Howe and H. Tan $[\mathrm{H}-\mathrm{T}, \S 3.3]$. This proof gives directly the best possible estimate for the operator norm of every radial measure supported on $S L_{2}(\mathbb{R}) \subset H$. Many more examples of the same phenomenon are provided by a systematic generalization of the arguments in $[\mathrm{H}-\mathrm{T}, \mathrm{Ch}$. V] due to H. Oh. First consider the following definition, introduced by G. A. Margulis [M2] :

Definition 5. Let $K$ and $H$ be closed subgroups of an lcsc group $G, K$ compact. $H$ is called $(G, K)$-tempered if for every unitary representation $\pi$ of $G$ without $G$-invariant unit vectors, and any $\pi(K)$-invariant unit vectors $v, w \in \mathcal{H}_{\pi}$, the matrix coefficient $|\langle\pi(h) v, w\rangle| \leq q(h)$, where $q \in L^{1}(H)$.

Many examples of $(G, K)$-tempered subgroups of a simple non-compact Lie group $G$ (where $K$ is maximal compact) are constructed in [M2] and [Oh]. We have

Proposition 4.1. Let $H$ be a $(G, K)$-tempered subgroup of $G=S L_{n}(\mathbb{R}), n \geq 3$ or $S p_{2 n}(\mathbb{R}), n \geq 2, K$ a maximal compact subgroup of $G$. Then the restriction to $H$ of any unitary representation $\pi$ of $G$ without $G$-invariant unit vectors satisfies $\left.\pi\right|_{H} \subset \infty \cdot \lambda_{H}$.

Proof. In [Oh, Prop. 3.4] It is shown that each $K$-finite matrix coefficient of an irreducible non-trivial unitary representations of $G$ is dominated on $A_{+}$by a constant multiple of a fixed positive function denoted $F_{G}$. The same proof applies without change to show that $K$-finite matrix coefficients in any unitary representation of $G$ without $G$-invariant unit vectors satisfy the same bound. In [Oh, Th. D] it is shown that when $H$ is $(G, K)$-tempered, $F_{G} \in L^{1}(H)$ (and 
conversely). It follows that $F_{G} \in L^{2}(H)$, and since $K$-finite matrix coefficient span a dense subset of the representation space, the Proposition follows from [H-T, Ch. V. 1.2.4].

\section{Remarks.}

1) Let $G$ be a simple non-compact Lie group of real rank $\geq 2, H$ any closed simple non-compact subgroup and $\pi$ a unitary representation of $G$ without $G$ invariant unit vectors. A sufficient condition that $\left.\pi\right|_{H}$ be weakly contained in $\lambda_{H}$ is that $\left.F_{G}\right|_{H} \in L^{2+\epsilon}(H)$ for every $\epsilon>0$. Here $F_{G}$ is defined in [Oh, Th. A], and the claim follows from [CHH, Th. 1].

2) Under the assumptions of Proposition 4.1 (or the previous remark), the estimate of the operator norm of absolutely continuous probability measures (or radial ones, or those supported on discrete subgroups of $H$ ) is that of the regular representation of $H$, and therefore the best possible. Hence in this case the best possible exponential-maximal inequalities and the fastest possible exponential rate of convergence is obtained for the averages considered in the previous sections, for example the sphere averages $\sigma_{n}$ on $H$, or on discrete wordhyperbolic subgroups of $H$. For further details on this phenomenon we refer to [N6].

3) To make the estimate of the operator norm in Theorem 1 (which are generally not optimal) explicit, one needs an estimate of the integer $n=n(X)$ such that $\pi_{0}^{\otimes n} \subset \infty \cdot \lambda_{G}$. For semi-simple Kazhdan groups $n=n(G)$ depends on $G$ only, and equals the least even integer $\geq p(G) / 2$, where $p(G)$ is calculated in $[\mathrm{Li}]$ and $[\mathrm{Oh}]$.

\subsection{Some further remarks and relevant references.}

1) As is well known (see e.g. [HRV, Prop. 1(6)]), given a (strongly continuous) unitary representation $\pi$ of an lcsc group $G$ and a probability measure $\mu$, if $\|\pi(\mu)\| \leq 1-\varepsilon$, then for any unit vector $v$,

$\varepsilon \leq 1-\|\pi(\mu) v\| \leq\|\pi(\mu) v-v\|=\left\|\int_{G}(\pi(g) v-v) d \mu(g)\right\| \leq \int_{G}\|\pi(g) v-v\| d \mu(g)$.

It follows that for at least one $g \in \operatorname{supp} \mu=Q,\|\pi(g) v-v\| \geq \varepsilon$. Hence, (see definition $3 \S 1.2),(Q, \varepsilon)$ are Kazhdan constants for the representation $\pi$. In other words, every estimate of the form $\|\pi(\mu)\| \leq 1-\varepsilon$ yields also Kazhdan constants. In particular, the estimates of $[\mathrm{H}-\mathrm{T}, \mathrm{Ch} . \mathrm{V}, \S 3.1]$ show that the spherical averages $s_{t}$ supported on $S L_{2}(\mathbb{R})$ and given by $s_{t}=m_{K} * \delta_{a_{t}} * m_{K}$ where $a_{t}=\operatorname{diag}\left(e^{t}, e^{-t}\right)$, satisfy the estimate $\left\|\pi_{0}\left(s_{t}\right)\right\| \leq \Xi_{2}\left(s_{t}\right) \leq C(1+t) \exp (-t)$ where $\pi_{0}$ is a unitary representation of any group $G \supset S L_{2}(\mathbb{R}) \ltimes \mathbb{R}^{2} \supset S L_{2}(\mathbb{R})$, without $S L_{2}(\mathbb{R}) \ltimes \mathbb{R}^{2}$ invariant unit vectors. Similarly, each of the norm estimates of the preceding sections determines Kazhdan constants for $G$.

Explicit Kazhdan constants for semi-simple groups were obtained originally from exponential decay estimates of matrix coefficients of $K$-finite vectors in irreducible representation of semi-simple groups $G$, due to M. Cowling [Co2] 
and R. Howe $[\mathrm{H}]$ (see $[\mathrm{H}$, remark a), p.311]). In particular these results of course provide estimates of the operator norm of any bi- $K$-invariant measure on $G$. For an elegant elementary derivation of such quantitative decay estimates for $S L_{n}(\mathbb{R}), n \geq 3$, as well as the corresponding explicit Kazhdan constants, we refer to [H-T, ch. V, $\S \S 3,4]$.

2) We note also the following fact, due to P. de la Harpe and A. Valette [HV] (see $\S 3.3$ for the notation), which computes Kazhdan constants for lattice subgroups :

Proposition 4.2. [HV, Ch. 3, Proof of Lemme 3] Let $G$ be a locally compact group, $\Gamma$ a lattice subgroup, normalize $m(G / \Gamma)=1$, and let $(\epsilon, Q)$ be a set of Kazhdan constants for $G$. Let $Y \subset G / \Gamma$ be any compact subset satisfying for every $k \in Q: m(Y \Delta k Y)<\frac{\epsilon^{2}}{2} m(Y)$. Let $s: G / \Gamma \rightarrow G$ be any measurable section which is bounded on compact sets, and $\alpha: G \times G / \Gamma \rightarrow \Gamma$ be the corresponding cocycle (see §3.3). Then if $L=\alpha\left(Q^{-1} \times Y\right) \subset \Gamma, L^{-1}$ and $\epsilon / 2$ are Kazhdan constants for $\Gamma$.

3) The spectral transfer principle was noted and applied by M. B. Bekka and A. Valette in [BV] and [B1, B2]. In particular, a simple alternative proof of some results due to $[\mathrm{C}-\mathrm{S}]$ is given in [B1]. In [B2] it is noted that in the action of a semi-simple Lie group $G$ in $L^{2}(G / \Gamma)$, integration is the unique finitely-additive mean invariant under the lattice subgroup $\Gamma$. This result relies on J. Rosenblatt's characterization of this property [Ro], namely that the unitary representation $\pi_{0}$ does not contain the trivial representation weakly. Together with the spectral transfer principle it follows that integration is the unique finitely-additive mean invariant under any lattice subgroup, in any measure-preserving ergodic action of $G$. A generalization of this fact can be found in [Sh], together with some further remarks regarding Kazhdan constants.

\subsection{Almost orthogonality.}

It is also possible to obtain ergodic theorems for (the averages of) a sequence of measures $\mu_{k}$, when the operator norms do not decay to zero at all. A sufficient condition is that the sequence $\pi\left(\mu_{k}\right) f=f_{k}$ forms an almost orthogonal sequence of functions (see [St]), in the sense that the decay of the mutual correlations satisfies $\left|\left\langle f_{k}, f_{m}\right\rangle\right| \leq C \exp (-\delta|k-m|)$, as the following standard result shows :

Theorem 4.3. (see [St, Cor. 2.4.1, Th. 3.7.2]) Let $a_{i}$ be positive constants, $a_{i} \uparrow \infty$. Let $f_{i}$ be a sequence of functions on a probability space, satisfying $\left|\left\langle f_{i}, f_{j}\right\rangle\right| \leq \rho_{|j-i|}\left\|f_{i}\right\|_{2}\left\|f_{j}\right\|_{2}$, where $0 \leq \rho_{i} \leq 1$ and $\sum_{i=1}^{\infty} \rho_{i}<\infty$.

(i) If $\sum_{k=1}^{\infty}(\log k)^{2} \frac{\left\|f_{k}\right\|_{2}^{2}}{a_{k}^{2}}<\infty$, then $\frac{1}{a_{N}} \sum_{k=0}^{N} f_{k}(x) \rightarrow 0$ for almost all $x \in X$,

(ii) In particular, if $0 \leq \rho_{k} \leq e^{-\delta k}, \delta>0$ and $\left\|f_{k}\right\|^{2} \leq M$, the choice $a_{k}=$ $\sqrt{k}(\log k)^{\frac{3}{2}+\varepsilon}$, yields $\left|\frac{1}{N+1} \sum_{k=0}^{N} f_{k}(x)\right| \leq C_{\epsilon}(x, f) \frac{(\log N)^{\frac{3}{2}+\epsilon}}{\sqrt{N}}$ for almost all $x \in X$ (and every $\epsilon>0$ ). 
We note the following sample consequences for a connected semi-simple Lie group $G$ with finite center and no compact factors. Let $K \subset G$ be a maximal compact subgroup, write $G=K \overline{A_{+}} K$, and let $a_{t}=\exp t H \in \overline{A_{+}}$, where $H \in \overline{\mathfrak{a}_{+}}$, $H \neq 0$. Consider again the operators defined by W. Veech $[\mathrm{V}]$ (see $\S 2.3$ ) given by $w_{t}=m_{K} * \delta_{a_{t}}$, and their adjoints $w_{t}^{*}=\delta_{a_{-t}} * m_{K}$. We will use the following estimate for bi- $K$-invariant matrix coefficients.

Theorem 4.4. (See [Co2, Proof of Cor. 2.4.3], [KM, Th. 2.4.3, Cor. 2.4.4], [KaSp, Th. 3.1].) Let $G$ be a connected semi-simple Lie group with finite center and no compact factors. If the representation $\pi$ satisfies $\pi^{\otimes n} \subset$ $\infty \cdot \lambda_{G}$ then for any two $K$-invariant unit vectors $v, w \in \mathcal{H},|\langle\rho(g) v, w\rangle| \leq$ $C \exp \left(-\frac{1}{M} d(e, g)\right)$, where $d$ is a left-invariant Riemannian distance on $G$ biinvariant w.r.t. $K$, and $C, M$ depend only on $n$ and $G$.

We can now formulate the following

Theorem 10. Let $G$ be a connected semi-simple Lie group with finite center and no compact factors. Assume $G$ acts on $X$, preserving the probability measure $m$, and $\pi_{0}^{\otimes n} \subset \infty \cdot \lambda_{G}$. For $0 \neq H \in \mathfrak{a}$ consider the averages $u_{t}=\delta_{\exp t H} * m_{K}$. Then for any $f \in L^{2}(X)$ (and every $\epsilon>0$ ),

$$
\left|\frac{1}{N+1} \sum_{k=0}^{N} \pi\left(u_{k}\right) f-\int_{X} f d m\right| \leq C(x, f) \frac{(\log N)}{\sqrt{N}}^{\frac{3}{2}+\epsilon} .
$$

In other words, $\frac{1}{n+1} \sum_{k=0}^{n} \pi\left(u_{k}\right)$ is a pointwise ergodic sequence in $L^{2}(X)$ with

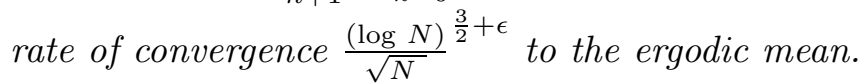

Proof. For $f \in L_{0}^{2}(X)$ and $f_{k}=\pi\left(u_{k}\right) f$, we have by Theorem 4.4 :

$$
\begin{gathered}
\left|\left\langle\pi\left(u_{t}\right) f, \pi\left(u_{s}\right) f\right\rangle\right|=\left|\left\langle\pi(\exp t H) \pi\left(m_{K}\right) f, \pi(\exp s H) \pi\left(m_{K}\right) f\right\rangle\right| \\
=\left|\left\langle\pi\left(m_{K}\right) \pi(\exp (t-s) H) \pi\left(m_{K}\right) f, \pi\left(m_{K}\right) f\right\rangle\right| \\
\leq C \exp \left(-\frac{1}{M} d(e, \exp (t-s) H)\right) \cdot\left\|\pi\left(m_{K}\right) f\right\|_{2}^{2} \\
\leq C \exp (-\delta|t-s|)\|f\|_{2}^{2} .
\end{gathered}
$$

Here $\delta>0$ since $d(e, \exp r H) \geq c|r|$ for some positive $c$, if $H \neq 0$. Hence the result follows from Theorem 4.3.

\section{Remarks.}

(1) Note that $\lim _{N \rightarrow \infty} \frac{1}{N+1} \sum_{k=0}^{N} \pi(\exp k H) f=\int_{X} f d m$ almost everywhere, by Birkhoff's theorem for the group $\mathbb{R}=\{\exp t H \mid t \in \mathbb{R}\}$. However, these averages converge to the ergodic mean arbitrarily slowly for some functions. This follows since $\left.\pi_{0}\right|_{\mathbb{R}} \cong \infty \cdot \lambda_{\mathbb{R}}$ (see [Co2, Cor. 2.4.3]) in this case. 
(2) Note also that $\frac{1}{N+1} \sum_{n=0}^{N} \pi\left(u_{n}\right)$ satisfies the strong maximal inequality in $L^{2}$, since already the averages $\frac{1}{N+1} \sum_{k=0}^{N} \pi(\exp k H)$ satisfy it.

Taking $\pi\left(u_{k}\right)=\pi(\exp (k H)) \pi\left(m_{K}\right)$ amounts to evaluating $\pi(\exp (k H))$ on $K$ invariant functions. More generally, it is possible to obtain a similar statement for any $C^{\infty}$-vectors $v, w \in C^{\infty}\left(\mathcal{H}_{\pi}\right)$, using the estimate :

$$
|\langle\pi(g) v, w\rangle| \leq C(v, w) \exp \left(-\frac{1}{M} d(e, g)\right)
$$

which holds provided $\pi^{\otimes n} \subset \infty \cdot \lambda_{G}$ (see [Co2, Proof of Cor. 2.4.3][KaSp, Th. $3.1][\mathrm{KM}])$. We therefore formulate the following result, whose proof is analogous to the proof of Theorem 10 :

Proposition 4.5. Let $G, X$ and $\pi$ be as in Theorem 10. Let $g_{k} \in G$ be a sequence satisfying $d\left(g_{i}, g_{j}\right) \geq \theta_{|i-j|}$ where $\sum_{k=1}^{\infty} e^{-\frac{1}{M} \theta_{k}}<\infty$. Then for any $C^{\infty}$ vector $f \in C^{\infty}\left(L^{2}(X)\right)$, we have (for any $\epsilon>0$ ) for almost every $x \in X$

$$
\left|\frac{1}{N+1} \sum_{k=0}^{N} \pi\left(g_{k}\right) f(x)-\int_{X} f d m\right| \leq C_{\varepsilon}(x, f) \frac{(\log N)}{\sqrt{N}}^{\frac{3}{2}+\epsilon} .
$$

Example. Take $g_{k}=g^{k}$, where $d\left(e, g^{k}\right) \geq c|k|$ for some positive $c$. Again note that by the previous remarks, the estimate of Prop. 4.5 cannot hold for all $f \in L^{2}(X)$.

\section{References}

[B1] M. B. Bekka, Restrictions of unitary representations to lattices and associated $C^{*}$ algebras, Funct. Analysis 143 (1997), 3-41.

[B2] - On uniqueness of invariant means, Proc. Amer. Math. Soc. (to appear).

[BV] M. B. Bekka and A. Valette, Lattices in semi-simple groups, and multipliers of group $C^{*}$-algebras, In: Recent advances in operator algebras (Orleans, 1992), Astérisque 232 (1995), 67-79.

[B-S] C. Bishop and T. Steger, Representation theoretic rigidity in $P S L(2, \mathbb{R})$, Acta. Math. 170 (1993), 121-149.

[Ca] A. P. Calderón, Ergodic theory and translation-invariant operators, Proc. Nat. Acad. Sci. U.S.A. 59 (1968), 349-353.

[C-W] R. R. Coifman and G. Weiss, Transference Methods in Analysis, CBMS Regional Conference Series in Math., 31, 1976..

[Coo] M. Coornaert, Mesures de Patterson-Sullivan sur le bord d'un space hyperbolique au sens de Gromov, Pacific J. Math, 159 (1993), 241-270.

[Co1] M. Cowling, The Kunze-Stein phenomenon, Annals of Math. 107 (1978), 209-234.

[Co2] Sur les coefficients des représentations unitaires des groupes de Lie simples, pp. 132-178, Lecture Notes in Mathematics , 739, Springer, Berlin, 1979.

[Co3] _ Harmonic analysis on semigroups, Ann. of Math 117 (1983), 267-283.

[Co4] _ Herz's "principe de majoration" and the Kunze-Stein phenomenon, UNSW preprint (1996). 
[C-H-H] M. Cowling, U. Haagerup, and R. Howe, Almost $L^{2}$ matrix coefficients, J. Reine. Angew. Math. 387 (1988), 97-110.

[C-S] M. Cowling and T. Steger, Irreducibility of restrictions of unitary representations to lattices, J. Reine. Angew. Math. 420 (1991), 85-98.

[Cl-St] J. L. Clerc and E. M. Stein, $L^{p}$-multipliers for non-compact symmetric spaces, Proc. Nat. Acad. Sci. U.S.A. 71 (1974), 3911-3912.

[C-V-J] P. A. Cherix, A. Valette, and P. Jolissaint, On spectra of simple random walks on one-relator groups, Pacific J. Math., 175 (1996), 417-438.

[E-M] A. Eskin and C. McMullen, Mixing, counting and equidistribution in Lie groups, Duke Math. J. 71 (1993), 143-180.

[Ey] P. Eymard, L'algébre de Fourier d'un groupe localement compact., Bull. Soc. Math. de France 92 (1964), 181-236.

[F-N] K. Fujiwara and A. Nevo, Maximal and pointwise ergodic theorems for word hyperbolic groups, Ergodic Theory Dynam. Systems 18 (1998), 1-16.

[G-V] R. Gangolli and V. S. Varadarajan, Harmonic analysis of spherical functions on read reductive groups, Ergeb. Math. Grenzgeb., Springer-Verlag, Berlin-New York, 1988.

[H-R-V] P. de la Harpe, A. G. Robertson and A. Valette, On the spectrum of the sum of generators of a finitely generated group, Israel J. Math 81 (1993), 65-96.

[HV] P. de la Harpe and A. Valette, Propriété (T) de Kazhdan pour les groupes localement compacts, Astérisque 175 (1989).

[He 2] S. Helgason, Groups and geometric analysis. Integral geometry, invariant differential operators, and spherical functions, Pure Appl. Math., 113, Academic Press, Inc., Orlando, Fla., 1984.

[Hz] C. S. Herz, Sur le phénomène de Kunze-Stein, C. R. Acad. Sci. Paris Sèrie A 271 (1970), 491-493.

[Hz1] The theory of p-spaces with an application to convolution operators, Trans. Amer. Math. Soc. 154, (1971), 69-82.

[H] R. Howe, On a notion of rank for unitary representations of the classical groups, Harmonic analysis and group representations, Liguori, Naples, 1982, pp. 223-331.

[H-T] R. Howe and E. C. Tan, Non-Abelian harmonic analysis (1992), Springer Verlag.

[J] P. Jolissaint, Rapidly decreasing functions in reduced $C^{*}$-algebras of groups, Trans. Amer. Math. Soc. 317 (1990), 167-196.

[Ka] D. A. Kazhdan, On a connection between the dual space of a group and the structure of its closed subgroups, Func. Anal. Appl. 1 (1967), 63-65.

[Ka-Sp] A. Katok and R. Spatzier, First cohomology of Anosov actions of higher rank abelian groups and applications to rigidity, I.H.E.S. Publ. Math. 79 (1994), 131-156.

$[\mathrm{K}-\mathrm{M}] \quad$ D. Y. Kleinbock and G. A. Margulis, Bounded orbits of non-quasi-unipotent flows on homogeneous spaces, Amer. Math. Soc. Transl. 171 (1996), 141-172.

[K-S] R. A. Kunze and E. M. Stein, Uniformly bounded representations and harmonic analysis of the $2 \times 2$ unimodular group, Amer. J. Math. 82 (1960), 1-62.

[Li] J.-S. Li, The minimal decay of matrix coefficients for classical groups, Harmonic analysis and its applications in China, vol. 327, Kluwer Academic Publishers, 1995, pp. $146-169$.

[M] G. A. Margulis, Discrete subgroups of semisimple Lie groups, A Series of Modern Surveys in Mathematics, vol. 17, Springer Verlag, 1991.

[M1] Discrete subgroups of motions of manifolds of non-positive curvature, Amer. Math. Soc. Transl 109 (1977), 33-45.

[M2] Existence of compact quotients of homogeneous spaces, measurably proper actions, and decay of matrix coefficients, Preprint (1997).

[M-N-S] G. A. Margulis, A. Nevo, and E. M. Stein, Analogs of Wiener's ergodic theorems for semi-simple Lie groups II, Submitted. 
[Mo] C. C. Moore, Exponential decay of correlation coefficients for geodesic flows, Group representations, ergodic theory, operator algebras, and mathematical physics (Berkeley, Calif., 1984), 163-181,, Math. Sci. Res. Inst. Publ., 6, Springer, New York-Berlin, 1987.

[N1] A. Nevo, Pointwise ergodic theorems for radial averages on simple Lie groups I, Duke Math. J. 76 (1994), 113-140.

[N2] , Pointwise ergodic theorems for radial averages on simple Lie groups II, Duke Math. J. 86 (1997), 239-259.

[N3] Harmonic analysis and pointwise ergodic theorems for non-commuting transformations, J. Amer. Math. Soc. 7 (1994), 875-902.

[N4] On discrete groups and pointwise ergodic theory, Proceedings of the conference on Harmonic Analysis and Discrete Potential Theory held at Cortona, Italy, June 1997, (M. Picardello and W. Woess, eds.), London Mathematical Society Series (to appear).

[N5] Spectral transfer and spectral multipliers, In preparation.

[N6] Lie groups and pointwise ergodic theory, In preparation.

[N-S1] A. Nevo and E. M. Stein, A generalization of Birkhoff's pointwise ergodic theorem, Acta Math. 173 (1994), 135-154.

[N-S2] A. Nevo and E. M. Stein, Analogs of Wiener's ergodic theorems for semi-simple Lie groups I, Ann. of Math., 145 (1997), 565-595.

[Oh] H. Oh, Tempered subgroups and reprersentations with minimal decay of matrix coefficients, Preprint (1997).

[Ro] J. Rosenblatt, Uniqueness of invariant means for measure-preserving transformations, Trans. Amer. Math. Soc. 265 (981), 623-636.

[Sh] Y. Shalom, Explicit Kazhdan constants for representations of semi-simple Lie groups and their lattices, Preprint, November (1997).

[S2] E. M. Stein, Harmonic analysis: real-variable methods, orthogonality, and oscillatory integrals, Princeton Mathematical Series, 43, Monographs in Harmonic Analysis, III, Princeton University Press, Princeton, NJ, 1993.

[St] W.F. Stout, Almost sure convergence (1974), Academic Press,, New York.

[T1] A. Templeman, Ergodic theorems for group actions 78 (1992), Kluwer Academic Publisher.

[V] W. A. Veech, Siegel measures, Preprint, June (1996).

[W] N. Wiener, The ergodic theorem, Duke Math. J. 5, 1-18 yr1939.

[Z] R. J. Zimmer, Ergodic theory and semi-simple groups, Monographs in Mathematics, 81, Birkhuser Verlag, Basel-Boston, Mass., 1984.

Dept. of Math., Technion-Israel Institute of Technology, 32000 Haifa, ISRAEL E-mail address: anevo@tx.technion.ac.il 